\title{
Analysis of Constant Deteriorating Inventory Management with Quadratic Demand Rate
}

\author{
Gobinda Chandra Panda ${ }^{1, *}$, Satyajit Sahoo ${ }^{1}$, Pravat Kumar Sukla ${ }^{2}$ \\ ${ }^{1}$ Dept of Mathematics ,Mahavir Institute of Engineering and Technlogy,Odisha, India \\ ${ }^{2}$ Panchay at Samiti College, Koksara, Kalahandi, Odisha, India
}

\begin{abstract}
This paper investigates inventory-production systems where items follow constant deterioration. The objective is to develop an optimal policy that minimizes total average cost. The quadratic demand technique is applied to control the problem in order to determine the optimal production policy, holding cost and cost of deterioration. Sensitivity analysis is conducted to study the effect of the cost parameters on the objective function.
\end{abstract}

Keywords Production, Inventory, Deterioration, Shortage, Quadratic demand

\section{Introduction}

The purpose of the present paper is to give a new dimension to the inventory literature on time varying demand patterns. Researchers have extensively discussed various types of inventory models with linear trend (positive or negative) in demand. The main Limitation in linear time-vary ing demand rate is that it implies a uniform change in the demand per unit time. This rarely happens in the case of any commodity in the market. In recent years, some models have been developed with a demand rate that changes exponentially with time. Demands for spare parts of new aeroplanes, computer chips of advanced computer machines, etc. decrease very rapidly with time. Some modellers suggest that this type of rapid change in demand can be represented by an exponential function of time. The present authors feel that an exponential rate of change in demand is extraord inarily high and the demand fluctuation of any commodity in the real market cannot be so high .A realistic approach is to think of accelerated growth (or decline) in the demand rate in the situations cited above and it can be best represented by a quadratic function of time. Thus, this paper has the scope of direct application in the very practical situations noted above.

Goods deteriorate and their value reduces with time. Electronic products may become obsolete as technology changes. Fashion tends to depreciate the value of clothing over time. Batteries die out as they age. The effect of time is even more critical for perishable goods such as foodstuff and cigarettes. The effect of deterioration and time/age is that the classical inventory model has to be readjusted K. Heng, J.

\footnotetext{
* Corresponding author:

pravatsukla2005@gmail.com (Pravat Kumar Sukla)

Published online at http://journal.sapub.org/ajor

Copyright (C) 2012 Scientific \& Academic Publishing. All Rights Reserved
}

Labban, R. Linn (1)

In general, deterioration is defined as decay, damage, spoilage, evaporation, obsolesce, pilferage, loss of utility or loss of marginal value of a commodity that results in decrease of us efulness from the original one. The decrease or loss of utility due to decay is usually a function of the on-hand inventory. It is reasonable note that a product may be understood to have lifetime, which ends when utility reaches zero.

The continuously decaying/deterioration of items is classified as age-dependent ongoing deterioration, and age-independent ongoing deterioration. Blood, fish, strawberry are some of the examples of the former while alcohol, gasoline and radioactive chemical and grain products are examples of the latter $\mathrm{H}$. Wee (4).

Haiping and Wang (7) developed an economic policy model for deteriorating items with time proportional demand. Donaldson (8) derived an analytical solution to the problems of obtaining the optimal number of replenishments and the optimal replenishment times of an EOQ model with a linearly time dependent demand pattern, over a finite time horizon. Zangwill (9) developed a discrete-in-time dynamic programming algorithm to solve an inventory model by allowing the inventory levels to be negative where the demand pattern is time dependent. Following

The approach of Donalds on (8), Murdeshwar (6,) Sahu and Sukla (10) has tried to derive an exact solution for a finite horizon inventory model to obtain the optimal number of replenishments, optimal replen is hment times and the optimal times at which the inventory level falls to zero, assuming the demand rate to be linearly time dependent and shortages. Hamid (3) presented a heuristic model for determining the ordering schedule when inventory items are subject to deterioration and demand changes linearly over time and obtained an optimal replenishment cycle length. Goswami and Chaudhuri (1) presented an EOQ model for deteriorating 
items with shortage and linear trend in demand. Bradshaw and Errol (2), published a paper in which they derived unbounded control policies for a class of linear time invariant production-inventory systems.

This paper investigates inventory-production systems where items follow constant deterioration. The objective is to develop an optimal policy that minimizes the cost associated with inventory and production rate. The quadratic demand technique is applied to control the problem in order to determine the optimal production policy. Sensitivity analysis is conducted to study the effect of the cost parameters on the objective function.

\section{Assumptions and Notations}

The following assumptions and notations have been used in developing the model.

(i) The demand rate is assumed to be $R(t)=a+b t+c t^{2}, a, b$ and $\mathrm{c}$ are constants. Such that $a>0, b>0, c>0$. Here a stands for the initial demand rate and $\mathrm{b}$ for the positive trend in demand.

(ii) The production rate Say $k=\gamma R(t)$, where $\gamma>1$. A fraction $\theta, 0<\theta<1$ of the on-hand inventory deteriorates per unit time.

(iii) The lead-time is zero and shortages are not allowed.

(iv) Unit holding cost $C_{1}$ per unit time and unit deterioration cost $C_{3}$ per unit time are known and constants.

(v) $C$ is the total average cost for the production cycle and $S$ is the stock level reached in the cycle.

(vi) The set up cost is not considered in this model because it is taken to be fixed for the whole cycle time.

(vii) Planning horizon is finite.

\section{Mathematical Formulation and Solution}

Let $q$ be the inventory level at any time $t \quad\left(0 \leq t \leq t_{2}\right)$. The differential equations governing the system in the interval $\left(0, t_{2}\right)$ are

$$
\begin{gathered}
\frac{d q}{d t}+\theta q=k-R(t), 0 \leq t \leq t_{1} \\
\frac{d q}{d t}+\theta q=-R(t), t_{1} \leq t \leq t_{2}
\end{gathered}
$$

The stock level initially is zero. Production begins just after $t=0$, continues up to $t=t_{1}$ and stops as soon as the stock level becomes $S$. Then the inventory level decreases due to demand and deterioration both till it becomes zero at $t=t_{2}$. The cycle then repeats itself. Our objective is to determine the optimum values of $S, C, t_{1}$ and $t_{2}$. The intensity of deterioration is very low in itially but it increases with time. However, it remains bounded for $t>>1$

Using the value of $R(t)$, the two equations (1) and (2) take the form

$$
\begin{aligned}
& \frac{d q}{d t}+\theta q=(\gamma-1)\left(a+b t+c t^{2}\right), 0 \leq t \leq t_{1} \\
& \text { and } \frac{d q}{d t}+\theta q=-\left(a+b t+c t^{2}\right), t_{1} \leq t \leq t_{2}
\end{aligned}
$$

The solution of equation (3) with initial conditions is

$$
q e^{\theta t}=(\gamma-1) \int_{0}^{t}(1+\theta t)\left(a+b t+c t^{2}\right) d t
$$

or

$$
q=(\gamma-1)\left(a t+\frac{b t^{2}}{2}+\frac{c t^{3}}{3}-\frac{a \theta t^{2}}{2}-\frac{b \theta t^{2}}{6}-\frac{c \theta t^{4}}{12}\right)(5)
$$

Neglecting the powers of $\theta$ greater than 1 .

Similarly, the solution of equation (4) also is (neglecting the powers of $\theta$ greater than 1)

$$
q \exp (\theta t)=C^{\prime}-\int\left(a+b t+c t^{2}\right) \exp (\theta t) d t
$$

or

$$
q=C^{\prime}(1-\theta t)+\left(-a t-\frac{b t^{2}}{2}-\frac{c t^{3}}{3}+\frac{a \theta t^{2}}{2}+\frac{b \theta t^{2}}{6}+\frac{c \theta t^{4}}{12}\right)
$$

For $t=t_{1}, q=S$

$$
\therefore S=C^{\prime}\left(1-\theta t_{1}\right)+\left(-a t_{1}-\frac{b t_{1}^{2}}{2}-\frac{c t_{1}^{3}}{3}+\frac{a \theta t_{1}^{2}}{2}+\frac{b \theta t_{1}^{2}}{6}+\frac{c \theta t_{1}^{4}}{12}\right)
$$

From (6) and (7) we get the relation

$$
q=S\left(1+\theta t_{1}-\theta t\right)+a\left(t_{1}-t+\frac{\theta t_{1}^{2}}{2}-\theta t_{1} t+\frac{\theta t^{2}}{2}\right)
$$




$$
+b\left(\frac{t_{1}^{2}}{2}-\frac{t^{2}}{2}+\frac{\theta t^{3}}{3}+\frac{\theta t^{3}}{6}-\frac{\theta t_{1}^{2}}{2}\right)+c\left(\frac{t_{1}^{3}}{3}-\frac{t^{3}}{3}+\frac{\theta t_{1}^{4}}{4}+\frac{\theta t^{4}}{12}-\frac{\theta t_{1}^{3} t}{3}\right)
$$

Using the condition $q=0$ for $t=t_{2}$ in equation (8), we get

$$
\begin{aligned}
S=a\left(t_{2}-t_{1}\right. & \left.+\frac{\theta t_{1}^{2}}{2}+\frac{\theta t_{2}^{2}}{2}-\theta t_{1} t_{2}\right)+b\left(\frac{t_{2}^{2}}{2}-\frac{t_{1}^{2}}{2}-\frac{\theta t_{1}^{3}}{6}+\frac{\theta t_{2}^{3}}{3}-\frac{\theta t_{1} t_{2}^{2}}{2}\right) \\
& +c\left(\frac{t_{2}^{3}}{3}-\frac{t_{1}^{3}}{3}+\frac{\theta t_{1}^{4}}{12}+\frac{\theta t_{2}^{4}}{4}-\frac{\theta t_{2}^{3} t_{1}}{3}\right)
\end{aligned}
$$

Now the average holding cost becomes

$$
\begin{gathered}
H(t)=\frac{C_{1}}{t_{2}}\left(\int_{0}^{t_{1}} q(t) d t+\int_{t_{1}}^{t_{2}} q(t) d t\right) \\
=\frac{C_{1}}{t_{2}}\left(\int_{0}^{t_{1}}(\gamma-1)\left(a t+\frac{b t^{2}}{2}+\frac{c t^{3}}{3}-\frac{a \theta t^{2}}{2}-\frac{b \theta t^{2}}{6}-\frac{c \theta t^{4}}{12}\right) d t\right. \\
+\int_{t_{1}}^{t_{2}}\left(S\left(1+\theta t_{1}-\theta t\right)+a\left(t_{1}-t+\frac{\theta t_{1}^{2}}{2}-\theta t_{1} t+\frac{\theta t^{2}}{2}\right)\right. \\
+b\left(\frac{t_{1}^{2}}{2}-\frac{t^{2}}{2}+\frac{\theta t^{3}}{3}+\frac{\theta t^{3}}{6}-\frac{\theta t_{1}^{2}}{2}\right)+c\left(\frac{t_{1}^{3}}{3}-\frac{t^{3}}{3}+\frac{\theta t_{1}^{4}}{4}+\frac{\theta t^{4}}{12}-\frac{\theta t_{1}^{3} t}{3}\right)
\end{gathered}
$$

Now substituting the value of $S$ from (9) and simplifying we get

$$
\begin{array}{r}
H(t)=\frac{C_{1}}{t_{2}}+a\left(\left(\frac{\gamma t_{1}^{2}}{2}-\frac{\gamma \theta t_{1}^{3}}{6}+\frac{t_{2}^{2}}{2}-t_{1} t_{2}+\frac{\theta t_{2}^{3}}{6}-\frac{\theta t_{2}^{2} t_{1}}{2}+\frac{\theta t_{2}^{2} t_{1}^{2}}{2}\right)\right. \\
+\frac{b}{6}\left(\frac{\gamma t_{1}^{3}}{6}-\frac{\gamma \vartheta t_{1}^{4}}{24}+\frac{t_{2}^{3}}{3}-\frac{t_{2}^{2} t_{1}}{2}+\frac{\theta t_{2}^{4}}{8}-\frac{\theta t_{2}^{3} t_{1}}{3}+\frac{\theta t_{1}^{3} t_{2}^{2}}{4}\right) \\
\left.+c\left(\frac{\gamma t_{1}^{4}}{12}-\frac{\gamma \theta t_{1}^{5}}{60}+\frac{t_{2}^{4}}{4}-\frac{\theta t_{2}^{3} t_{1}}{3}+\frac{\theta t_{2}^{5}}{10}-\frac{\theta t_{2}^{4} t_{1}}{4}+\frac{\theta t_{2}^{3} t_{1}^{2}}{6}\right)\right)
\end{array}
$$

The average cost due to deterioration in the total cycle time is

$$
\begin{array}{r}
d(t)=\frac{C_{3}}{t_{2}}\left[\gamma \int_{0}^{t_{1}}\left(a+b t+c t^{2}\right) d t-\int_{0}^{t_{2}}\left(a+b t+c t^{2}\right) d t\right] \\
=\frac{C_{3}}{t_{2}}\left[a\left(\gamma t_{1}-t_{2}\right)+\frac{b}{2}\left(\gamma t_{1}^{2}-t_{2}^{2}\right)+\frac{c}{3}\left(\gamma t_{1}^{3}-t_{2}^{3}\right)\right]
\end{array}
$$

From (10) and (11) the total average cost of the inventory I

$$
\begin{gathered}
C=\frac{C_{1}}{t_{2}}\left(\frac{a}{6}\left(\left(3 \gamma t_{1}^{2}-\gamma \theta t_{1}^{3}+3 t_{2}^{2}-6 t_{1} t_{2}+\theta t_{2}^{3}-3 \theta t_{2}^{2} t_{1}+3 \theta t_{2} t_{1}^{2}\right)\right)\right. \\
+\frac{b}{24}\left(4 \gamma t_{1}^{3}-\gamma \theta t_{1}^{4}+8 t_{2}^{3}-12 t_{2}^{2} t_{1}+3 \theta t_{2}^{4}-8 \theta t_{2}^{3} t_{1}+6 \theta t_{1}^{2} t_{2}^{2}\right) \\
\left.+\frac{c}{12}\left(5 \gamma t_{1}^{4}-\gamma \theta t_{1}^{5}+15 t_{2}^{5}-20 \theta t_{2}^{3} t_{1}+6 \theta t_{2}^{5}-15 \theta t_{2}^{4} t_{1}+10 \theta t_{2}^{3} t_{1}^{2}\right)\right)
\end{gathered}
$$




$$
+\frac{C_{3}}{t_{2}}\left[a\left(\gamma t_{1}-t_{2}\right)+\frac{b}{2}\left(\gamma t_{1}^{2}-t_{2}^{2}\right)+\frac{c}{3}\left(\gamma t_{1}^{3}-t_{2}^{3}\right)\right]
$$

By putting $t_{1}=z t_{2}$ (where $0<z<1$ ) in equation (12), we get

$$
\begin{gathered}
C=\frac{C_{1}}{t_{2}}\left(\frac{a}{6}\left(3 \gamma z^{2} t_{2}^{2}-\gamma \theta t_{2}^{3} z^{3}+3 t_{2}^{2}-6 t_{2}^{2} z+\theta t_{2}^{3}-3 \theta t_{2}^{3} z+3 \theta t_{2}^{3} z^{2}\right)\right. \\
+\frac{b}{24}\left(4 \gamma z^{3} t_{2}^{3}-\gamma \theta z^{4} t_{2}^{4}+8 t_{2}^{3}-12 t_{2}^{3} z+3 \theta t_{2}^{4}-8 \theta t_{2}^{4} z+6 \theta t_{2}^{4} z^{2}\right) \\
\left.+\frac{c}{12}\left(5 \gamma z^{4} t_{2}^{4}-\gamma \theta z^{5} t_{2}^{5}+15 t_{2}^{4}-20 \theta t_{2}^{4} z+6 \theta t_{2}^{5}-15 \theta t_{2}^{5} z+10 \theta t_{2}^{5} z^{2}\right)\right) \\
+\frac{C_{3}}{t_{2}}\left[a\left(\gamma z t_{2}-t_{2}\right)+\frac{b}{2}\left(\gamma z^{2} t_{2}^{2}-t_{2}^{2}\right)+\frac{c}{3}\left(\gamma z^{3} t_{2}^{3}-t_{2}^{3}\right)\right]
\end{gathered}
$$

For calculating the optimum value of $C$ we differentiate it partially with respect to $t_{2}$ and equate them to zero. Thus we get the following equation:-

$$
\begin{gathered}
\frac{d C}{d t_{2}}=C_{1}\left(\frac{a}{6}\left(3 \gamma z^{2}-2 \gamma t_{2} z^{3}+3-6 z+2 \theta t_{2}-6 \theta t_{2} z+6 \theta t_{2} z^{2}\right)\right. \\
+\frac{b}{24}\left(8 \gamma z^{3} t_{2}-3 \gamma \theta z^{4} t_{2}^{2}+16 t_{2}-24 t_{2} z+9 \theta t_{2}^{2}-24 \theta t_{2}^{2} z+18 \theta z^{2} t_{2}^{2}\right) \\
\left.+\frac{c}{60}\left(15 \gamma z^{4} t_{2}^{2}-4 \gamma \theta z^{5} t_{2}^{3}+45 t_{2}^{2}-60 \theta t_{2}^{2} z+24 \theta t_{2}^{3}-60 \theta t_{2}^{3} z+40 \theta t_{2}^{3} z^{2}\right)\right) \\
+C_{3}\left[\frac{b}{2}\left(\gamma z^{2}-1\right)+\frac{2 c}{3}\left(\gamma z^{3}-1\right) t_{2}\right]
\end{gathered}
$$

This equation gives us the optimum value of $t_{2}$ which, when substituted equation (13), give the total average cost, provided $\frac{d^{2} C}{d t_{2}^{2}}>0$. Equation (14) is highly non-linear in $t_{2}$ and cannot be solved analytically. This equation, therefore, can be solved by some suitable numerical method like Newton-Raphson, and optimal value of $t_{2}$ can be obtained. This optimal value of $t_{2}$ gives the minimum cost of the system in question. We have solved this equation on computer for a set of values of the parameters with the help of Newton-Raphson method. A numerical example is given below as an illustration.

\subsection{Example-1}

Let $\theta=0.02, \mathrm{r}=2.0, \mathrm{z}=0.7, \mathrm{C}_{1}=5.0, \mathrm{C}_{3}=60, \mathrm{a}=200, \mathrm{~b}=40$, $\mathrm{c}=10$ in suitable units.

The solution for optimal values of $t_{1}$ and $t_{2}$ is $\mathrm{t}_{1}^{*}=1.5534, t_{2}^{*}=14.0345$, which gives minimum average cost $\mathrm{C}^{*}=10395.3646$

Following are a number of tables representing the optimal values of $t_{1}, t_{2}$ and $C$ as also the no-production interval $t_{2}-t_{1}$.

\section{Sensitivity Analysis}

We have discussed the effects of the different parameters.

(i) Increase in the value of $\gamma$ decreases the value of $t_{1}$, $t_{2}$ and $\mathrm{C}$.

(ii) Increase in the value of the parameter $\theta$, decrease the values of $t_{1}, t_{2}$ and $\mathrm{C}$.

(iii) Increase in the value of holding $\cos t C_{1}$ increases the value of the cost $t_{1}, t_{2}$ and decreases the value of $\mathrm{C}$.

(iv) Increase in the value of deterioration cost $C_{3}$ increases the value of the $\cos t t_{2}$ and $\mathrm{C}$. However the values of $t_{1}$ decrease. Increase in the value of a, decreases the value of $\mathrm{C}, t_{1}, t_{2}$. Increase in the value of $\mathrm{b}$, increases the values of $t_{1}, t_{2}$ and $\mathrm{C}$.

(v) Increase in the value of $\mathrm{c}$, decreases the value of C, $t_{1}$, $t_{2}$.

(vi) Keeping these variations in mind of the decision maker of the inventory system can control the parameters so as to optimize the objective function. The decision maker may control particularly the holding cost and the cost of deterioration for minimizing the total average cost. 
Table 1. Variations in parameters

\begin{tabular}{|c|c|c|c|c|c|}
\hline & Parameter & $t_{1}$ & $t_{2}$ & $\mathrm{C}$ & $t_{2}-t_{1}$ \\
\hline \multirow{5}{*}{$\theta$} & 0.005 & 1.5880 & 14.8152 & 10733.5044 & 13.2272 \\
\hline & 0.010 & 1.5828 & 14.7280 & 10677.6608 & 13.1452 \\
\hline & 0.015 & 1.5785 & 14.6057 & 10534.0876 & 13.0272 \\
\hline & 0.020 & 1.5534 & 13.8345 & 10395.3646 & 12.2811 \\
\hline & 0.025 & 1.5732 & 12.3273 & 10165.5343 & 11.7541 \\
\hline \multirow{5}{*}{ r } & 1.3 & 13.0202 & 26.2543 & 20546.0034 & 13.2341 \\
\hline & 1.5 & 9.7654 & 21.3454 & 17861.1234 & 11.5800 \\
\hline & 1.7 & 5.4560 & 16.7234 & 15332.2341 & 11.2674 \\
\hline & 1.9 & 2.8776 & 13.5431 & 12546.2341 & 10.6655 \\
\hline & 2.1 & 0.8834 & 11.2396 & 08537.7582 & 10.3562 \\
\hline \multirow{5}{*}{$\mathrm{a}$} & 190 & 2.8854 & 16.4725 & 12164.0551 & 13.5871 \\
\hline & 200 & 1.6043 & 14.7843 & 10633.6035 & 13.1800 \\
\hline & 210 & 1.4872 & 14.3172 & 09558.8551 & 12.8300 \\
\hline & 220 & 0.6623 & 12.9956 & 07440.6742 & 12.3333 \\
\hline & 230 & 0.2547 & 12.5427 & 04786.2316 & 12.2880 \\
\hline \multirow{5}{*}{ b } & 35 & 3.1437 & 4.4910 & 6689.7036 & 1.3473 \\
\hline & 40 & 3.2827 & 4.6896 & 6706.7988 & 1.4069 \\
\hline & 45 & 3.4539 & 4.9342 & 6726.3891 & 1.4803 \\
\hline & 50 & 3.6927 & 5.2754 & 6749.9902 & 1.5827 \\
\hline & 55 & 4.1103 & 5.8719 & 6782.2915 & 1.7616 \\
\hline \multirow{5}{*}{$\mathrm{c}$} & 8 & 3.6306 & 5.1867 & 6868.5703 & 1.5561 \\
\hline & 10 & 3.2827 & 4.6896 & 6706.7988 & 1.4069 \\
\hline & 12 & 2.3296 & 3.3280 & 6662.1054 & 0.9984 \\
\hline & 14 & 1.8543 & 2.6490 & 6600.9335 & 0.7947 \\
\hline & 16 & 1.5458 & 2.2083 & 6543.3886 & 0.6625 \\
\hline \multirow{4}{*}{$\mathrm{C}_{1}$} & 5 & 1.4480 & 14.2350 & 10722.5432 & 12.7870 \\
\hline & 6 & 2.3365 & 14.3956 & 10621.2031 & 12.0591 \\
\hline & 7 & 2.7798 & 13.6065 & 10543.1232 & 10.8267 \\
\hline & 8 & 2.8134 & 12.8939 & 10327.1212 & 10.0805 \\
\hline \multirow{5}{*}{$\mathrm{C}_{3}$} & 40 & 2.7257 & 12.8938 & 6576.6791 & 10.1681 \\
\hline & 50 & 2.2827 & 13.6896 & 6788.7988 & 11.4069 \\
\hline & 60 & 1.9006 & 13.8152 & 8200.9125 & 11.9146 \\
\hline & 70 & 1.7526 & 14.218 & 8558.0664 & 13.4654 \\
\hline & 80 & 1.0350 & 15.0500 & 1078.3515 & 14.0150 \\
\hline
\end{tabular}

\section{Conclusions}

In this article, a deterministic inventory model has been proposed for deteriorating item with quadratic demand rate, where shortages are not allowed. The goal of the paper is to incorporate the deterioration phenomenon together into an inventory model over a finite planning horizon. This paper investigates inventory-production systems where items follow constant deterioration. The objective is to develop an optimal policy that minimizes total average cost. The quadratic demand technique is applied to control the problem in order to determine the optimal production policy, holding cost and cost of deterioration.

\section{REFERENCES}

[1] Goswami, K. Chaudhuri, an EOQ model for deteriorating items with shortages and a linear trend in demand, J. Opl. 
Res. Soc. 42 (1991) 1105-1110.

[2] A. Bradshaw, Y. Erol, Control policies for production inventory systems with bounded input, Int. J. Sys. Sci. 11 (1980) 947-959.

[3] B. Hamid, Replenishment schedule for deteriorating items with time proportional demand. J. Oper. Res. Soc.40 (1989) 75-81.

[4] H. Wee, Economic production lot size model for deteriorating items withpartial back ordering, Comput. Ind. Eng. 24 (1993) 449-458.

[5] K. Heng, J. Labban, R. Linn. An order level for deteriorating items with partial back ordering, Comput. Ind. Eng. 20 (1991) 187-197

[6] T.M. Murdeshwar Inventory replenishment policy for linearly increasing demand considering shortages- an optimal solution. J.OplRes.Soc.39, (1988) 687692

[7] U. Haiping, H. Wang, An economic ordering policy model for deteriorating items with time proportional demand, Eur. J. Oper. Res. 46 (1990) 21-27.

[8] W.A. Donaldson Inventory replenishment policy for a linear trend in demand- an analy tical solution. Opl Res. Q. 28,(1977) 663-670.

[9] W.I Zangwill (1966) A deterministic multi-period production scheduling model with backlogging. Mgmt. Sci. 13, (1966) 105-119.

[10] S.K.Sahu ,P.K.Sukla,A note for weibul deteriorating model with time varying demand and partial back-ordering.Acta Cienia Indica,vol,xxxiv M,No,4,(2008) 1673-1670. 